\title{
IONIC CONDUCTIVITY AND LOW FREQUENCY DISPERSION IN HEXAGONAL SILVER IODIDE**
}

\author{
G. COCHRANE $\dagger$ and N. H. FLETCHER \\ Department of Physics, University of New England, Armidale, N.S.W. Australia
}

(Received 29 September 1969; in revised form 6 April 1971)

\begin{abstract}
Measurements are reported for the temperature variation of ionic conductivity in single crystals of hexagonal silver iodide. Both nominally pure and $\mathrm{Cu}^{2+}$ doped crystals were studied and analysis of the measurements for conduction both parallel and normal to the $c$-axis leads to a formation energy for Frenkel defects of $0.60 \mathrm{eV}$, activation energies for diffusion of $\mathrm{Ag}^{+}$interstitials parallel and normal to the $c$-axis of 0.61 and $0.29 \mathrm{eV}$ respectively and corresponding activation energies for $\mathrm{Ag}^{+}$vacancies of 0.50 and $0.39 \mathrm{eV}$. Similar measurements performed on thin films of $\mathrm{AgI}$ grown expitaxially on mica show varying behaviour but the activation energy for conduction is about 0.4 $\mathrm{eV}$, which agrees with the value expected from the single-crystal results if the conductivity is vacancy controlled.

Dielectric measurements in the frequency range $10-10^{5} \mathrm{~Hz}$ at temperatures between 193 and $340^{\circ} \mathrm{K}$ show anomalous dispersion peaks at about $10^{4} \mathrm{~Hz}$ for temperatures above $300^{\circ} \mathrm{K}$ which are tentatively interpreted in terms of trapped Frenkel defects and interfacial polarization. At temperatures below about $290^{\circ} \mathrm{K}$ a series of sharp resonant absorptions is observed whose origin is uncertain. The static dielectric constant approaches the value $6 \cdot 8 \pm 0 \cdot 2$ at temperatures below $-40^{\circ} \mathrm{C}$.
\end{abstract}

\section{INTRODUCTION}

THE ELECTRICAL and optical properties of the silver halides have received considerable attention over the past few decades; much of this interest has, however, been centred around silver chloride and silver bromide, a circumstance which can be attributed to the obvious commercial application and relative ease of obtaining large, pure, single crystals of these materials. A recent literature survey [1] revealed only one publication dealing with measurements of the electrical properties and none dealing with the optical properties of single crystals of AgI. It is proposed to present in this and following papers the results of a preliminary investigation on the optical and electrical properties of thin layers and single crystals of hexagonal silver iodide.

At atmospheric pressure silver iodide has been reported as trimorphic [2,3,4] with three further polymorphs appearing at ele-

\footnotetext{
*Based on a thesis submitted by G. Cochrane for the Ph.D. degree of the University of New England.

†Present Address: Royal Military College, Duntroon, A.C.T., Australia.
}

vated pressures[5]. Of those occurring at atmospheric pressure, two modifications coexist below a first order phase transition at about $147^{\circ} \mathrm{C}$, namely a cubic sphaleritetype structure, designated $\gamma \mathrm{AgI}$, and a hexagonal wurtzite-type structure designated $\beta A g I$. The stability regions of the $\gamma$ and $\beta$ modifications have created considerable controversy.

Some authors [3] give $\gamma \mathrm{AgI}$ as the stable phase at room temperature and $\beta \mathrm{AgI}$ as only metastable, while other authors $[2,4]$ give the reverse. Majumdar and Roy [6] doubt if $\gamma \mathrm{AgI}$ even exists. It is generally agreed from X-ray diffraction studies that nearly all powder specimens of $\mathrm{AgI}$ at room temperature and atmospheric pressure consist of a mixture of the cubic and hexagonal phases in varying proportions depending on the method of preparation [7]. The original conjecture that $\beta \mathrm{AgI}$ is only metastable below $135^{\circ} \mathrm{C}$ was put forward by Bloch and Moller [3] in 1931 and this has since been quoted (but apparently not verified) by several authors. The results of Burley[2] and of 
Schneer and Whiting[4] show that $\gamma$ AgI undergoes a transformation to $\beta \mathrm{AgI}$ at temperatures as low as $90^{\circ} \mathrm{C}$, while the reverse transition cannot be brought about below $147^{\circ} \mathrm{C}$ by thermal equilibration. An analysis of the experimental evidence suggests that the conclusions drawn by Bloch and Moller[3] are incorrect.

Unless special precautions are taken, the usual methods adopted for preparing AgI specimens suffer from several disadvantages which are often overlooked. These can be summarized as follows.

\section{Powder compacts}

The method of preparation is adequately covered in papers by Corrin and Storm [8] and by Jost and Oel[9]. A precaution which must be observed to maintain stoichiometry is the prevention of photolysis and consequent loss of iodine. Another disadvantage of powder compacts is that, if the compaction pressure exceeds about 100 bar, then, according to Burley [2], the final specimen will be predominantly $\gamma \mathrm{AgI}$, irrespective of the original proportions of $\beta \mathrm{AgI}$ and $\gamma \mathrm{AgI}$.

\section{Solidification from the melt}

Burley [2] observed that specimens obtained by quenching from the melt showed a predominance of the sphalerite structure, while those cooled slowly had the wurtzite structure. A difficulty always present when working with the melt is its tendency to decompose giving metallic silver and elemental iodine, again resulting in non-stoichiometric specimens.

\section{Thin films}

Layers deposited on a substrate have been used almost exclusively for optical work. A summary of thin film preparation is given elsewhere $[1,10]$. The major difficulty is to prepare stoichiometric layers of a predetermined phase type.

\section{Single crystals}

To facilitate the interpretation of results it is usually desirable to work with specimens cut from single crystals. Recently, several methods have been described for growing single crystals of $\beta \mathrm{AgI}$ large enough to permit the undertaking of electrical and optical experiments. Two of the methods rely on slow diffusive dilution of either a saturated AgI-KI[11] solution or a AgI-HI[12] solution, while a third[13] method relies on convective circulation of a AgI-KI solution between two chambers held at different temperatures. In addition to the above a silica gel method has been described by Halberstadt [14].

\section{IONIC CONDUCTIVITY MEASUREMENTS}

The general theory of the electrical conductivity of ionic crystals, formulated in terms of the motion of Frenkel or Schottky defects, is well established[15]. In the case of pure $\beta \mathrm{AgI}$ the conductivity mechanism is generally agreed to involve the motion of Frenkel defects, while transport measurements[16] have established that only the $\mathrm{Ag}^{+}$ions contribute appreciably to the electric current, both $\mathrm{I}^{-}$and electronic currents being negligible. Thus interstitial silver ions and their associated vacant lattice sites are primarily responsible for the conductivity.

Following an analysis given by Lidiard [15], the ionic conductivity results are presented by plotting $\ln (\sigma T)$ in terms of $T^{-1}$. In general the graph consists of two approximately linear segments. The upper segment extends from the melting point, or the highest temperature of stability of the solid phase of interest, to some lower temperature which is determined by the purity and perfection of the crystal. Normally, this region is an intrinsic property of the substance and measurements are quite reproducible. The low temperature conductivity, known as the extrinsic or structure sensitivity region, always displays a smaller slope. The magni- 
tude of the conductivity in this region depends principally on the nature and magnitude of the impurity content and the thermal history of the specimen.

The complete ionic conductivity curve is therefore roughly a superposition of two lines, represented by

$$
\sigma T=A_{1} \exp \left(-E_{1} / k T\right)
$$

for the high temperature or intrinsic region and

$$
\sigma T=A_{2} \exp \left(-E_{2} / k T\right)
$$

for the low temperature or extrinsic region.

In the high-temperature regime the Frenkel defects are generated intrinsically with a formation energy $W$ and the activation energy for conductivity can be written

$$
E_{1}=\frac{1}{2} W+U
$$

where $U$ is the activation energy for diffusion of an interstitial ion or of a vacancy, whichever is more mobile. In the low temperature regime the interstitial ions or vacancies are provided by the impurities and imperfections in the crystal, so that their concentration does not depend upon temperature and we can write

$$
E_{2}=U
$$

It is thus generally necessary to make measurements on crystals containing enough impurity that both linear regions can be adequately measured if we wish to determine both $W$ and $U$. Alternatively, information about $U$ can be obtained from self-diffusion studies using radioactive tracers [15], while an estimate of $W$ can be made from specific heat measurements [17].

\subsection{Experimental}

Single crystal specimens were grown from a HI-AgI solution as described by Lakatas and Lieser[12] or alternatively from a KI-AgI solution as described by Cochrane[11]. Spectrochemical analysis showed the only detectable impurities to be traces of potassium and sodium, while a flame photometer test set the potassium impurity at about $20 \mathrm{ppm}$ for crystals grown from KI-AgI solution. However, no differences were observed in the ionic behaviour of crystals obtained by the two methods. Plate-like specimens 0.27 to $0.88 \mathrm{~mm}$ thick were prepared by cleaving single crystals perpendicular to the $c$-axis with a razor blade. A specimen then had three electrodes applied to the freshly cleaved faces by evaporating silver through suitable masks. The lower specimen surface was completely coated to form one electrode while the upper surface had a central electrode, $0.088 \mathrm{~cm}$ in dia., concentrically surrounded by a guard electrode. An alternative arrangement, which gave identical results, was to use longer sections $(1.0$ to $4.0 \mathrm{~mm})$ of prismshaped crystals with each end face completely coated to form an electrode. For these specimens, in addition to silver, colloidal graphite (Aquadag) was also used as an electrode material. Since unwanted strains can readily be introduced into $\mathrm{AgI}$ specimens, no attempt was made to grind or alter the shape of the hexagonal cross-section. The samples were mounted in specimen holders fitted with beryllium copper contacts adjusted for the minimum contact pressure necessary to give noise-free behaviour.

For thin-film measurements, layers of $\beta A g I$ were prepared by condensing silver on a mica substrate, held at room temperature in the presence of an iodine atmosphere. Electron diffraction studies [10] have shown that the films used were well oriented with the stacking direction normal to the substrate. Electrical contact to the film was made through silver or colloidal graphite electrodes deposited on the mica surface prior to the commencement of evaporation. The thickness of the films was measured by an optical interference method similar to that described by Tolansky [18]. 
All conductivity measurements were made under vacuum conditions with a pressure of about $10^{-4}$ torr. The specimen temperature was measured with a copper-constantan thermocouple clamped adjacent to and in a similar manner to the specimen. The specimen temperature was controlled to $\pm 0 \cdot 1^{\circ} \mathrm{C}$ but quoted absolute temperatures are only accurate to $\pm 2^{\circ} \mathrm{C}$.

All the conductivity measurements were made using a simple or a guarded twoelectrode system as described and used either direct current or a low frequency $(0.5$ to $5 \mathrm{~Hz})$ square wave. When using direct currents there was some evidence of the growth of silver dendrites from the electrodes, particularly for thin-film specimens at elevated temperatures, however, this was minimized by the use of very small currents $\left(10^{-8}\right.$ to $\left.10^{-12} \mathrm{~A}\right)$ and by the limitation of measurement times to the minimum required to achieve stability.

\subsection{Results}

Initially current-voltage measurements were made on the longer sections of prismatic crystals to verify that the electrodes were truly ohmic. A typical set of room temperature results for a crystal with a pair of Aquadag electrodes is shown in Fig. 1. The initial curve, 1, was only obtained for a specimen which had not previously had a voltage applied across it. After measurements had been made at higher voltages, curve 1 could not be reproduced and the measurements followed curve 2 which was completely reproducible.

For temperatures above $260^{\circ} \mathrm{K}$ and below the $420^{\circ} \mathrm{K}$ transition point, silver electrodes were found to be completely ohmic in behaviour. Below $260^{\circ} \mathrm{K}$ silver electrodes exhibited ohmic behaviour only when the measuring voltage exceeded about one volt, below this current readings were very erratic. With thin-film specimens both silver and Aquadag electrodes exhibited ohmic behaviour.

Temperature dependence of the ionic

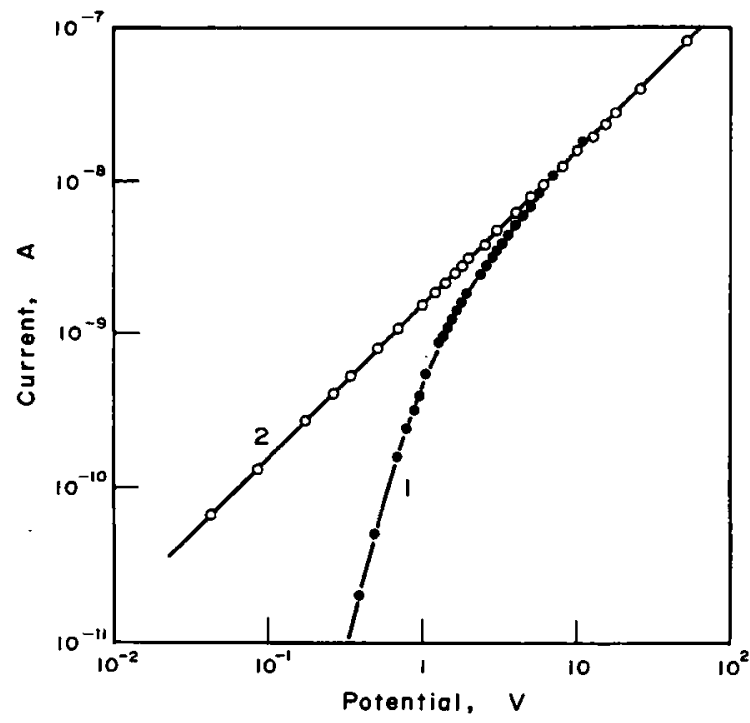

Fig. 1. Typical room temperature current-voltage characteristics for a single crystal specimen with colloidal graphite (Aquadag) electrodes. See text for explanation of curve 1 and curve 2 .

conductivity for over 30 single crystal specimens was measured for a current flow in the direction of the $c$-axis. The results are summarized in Fig. 2(a).

In order to resolve the activation energy $E$ into the two components $U$ and $W$, attempts were made to grow crystals containing impurity cations. The required impurity in the form of the iodide was added to the KI-AgI growing solution. With the exception of $\mathrm{Cu}^{2+}$ ions, subsequent analysis either by $\mathrm{X}$-ray fluorescence or atomic absorption failed to detect any traces of impurity. Atomic absorption measurements set the copper impurity at $0.5 \pm 0.1$ per cent by weight, although not all of this copper may be present as $\mathrm{Cu}^{2+}$. Visibly the copper-doped crystals did not appear any different from pure $\beta \mathrm{AgI}$; however, when attempts were made to cleave a prismshaped crystal, it was noticeably more brittle and failed to cleave cleanly. Only one specimen containing $\mathrm{Cu}^{2+}$ ions proved sufficiently large for testing, the results for this specimen with a current flow in the c-axis direction are shown in Fig. 2(a).

Figure 3 shows the variation of ionic 


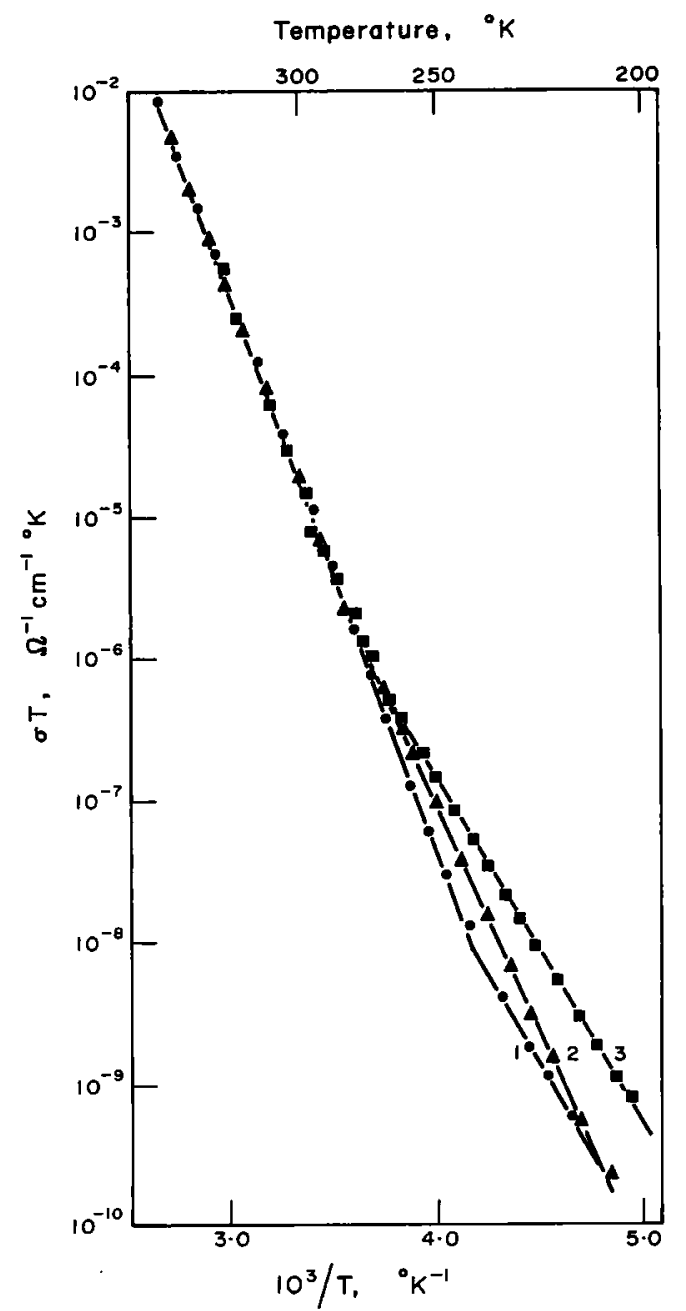

Fig. 2(a). Temperature dependence of the ionic conductivity of single crystals of $\beta \mathrm{AgI}$ with current flow parallel to the $c$-axis. (1) pure crystal with graphite electrodes;

(2) pure crystal with silver electrodes and (3) crystal containing about $1 / 2$ per cent by weight of $\mathrm{Cu}^{2+}$.

conductivity with temperature for a representative selection from over 40 thin-film specimens tested, ranging in thickness from 100 to $5000 \AA$. Apart from the measurement of film thickness, other experimental details were similar to those described for singlecrystal specimens.

\subsection{Discussion of ionic conductivity results}

Measurements of the type reported show that metallic silver provides a good ohmic

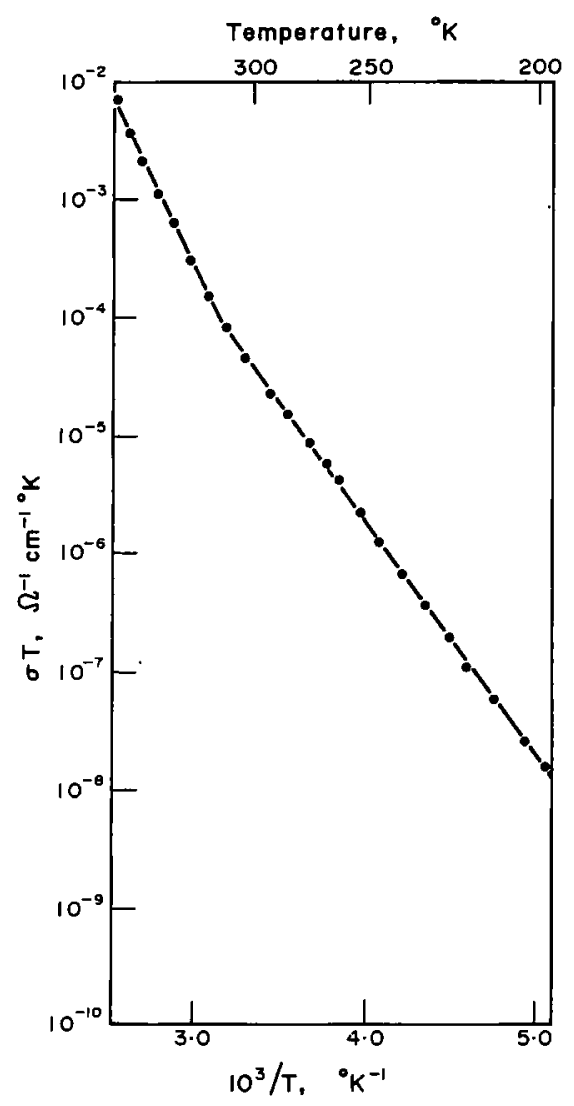

Fig. 2(b). Temperature dependence of the ionic conductivity of single crystals of $\beta \mathrm{AgI}$ with graphite electrodes and current flow perpendicular to the $c$-axis.

contact to $\beta$ AgI at temperatures above $260^{\circ} \mathrm{K}$. Colloidal graphite electrodes are initially non-ohmic but achieve ohmic behaviour after passage of an alternating current for a time at a reasonably high applied potential. Prolonged application of an alternating potential leads to growth of silver dendrites from both carbon electrodes; thus it is reasonable to assume that the conditioning process involves electrolytic build-up of silver so that the system becomes effectively |graphite $|\mathrm{Ag}| \mathrm{AgI}|\mathrm{Ag}|$ graphite|. With both electrode systems, then, we make the reasonable assumption that contact potential differences are negligible compared with $10 \mathrm{~V}$, the order of the potentials used in the measurements. 


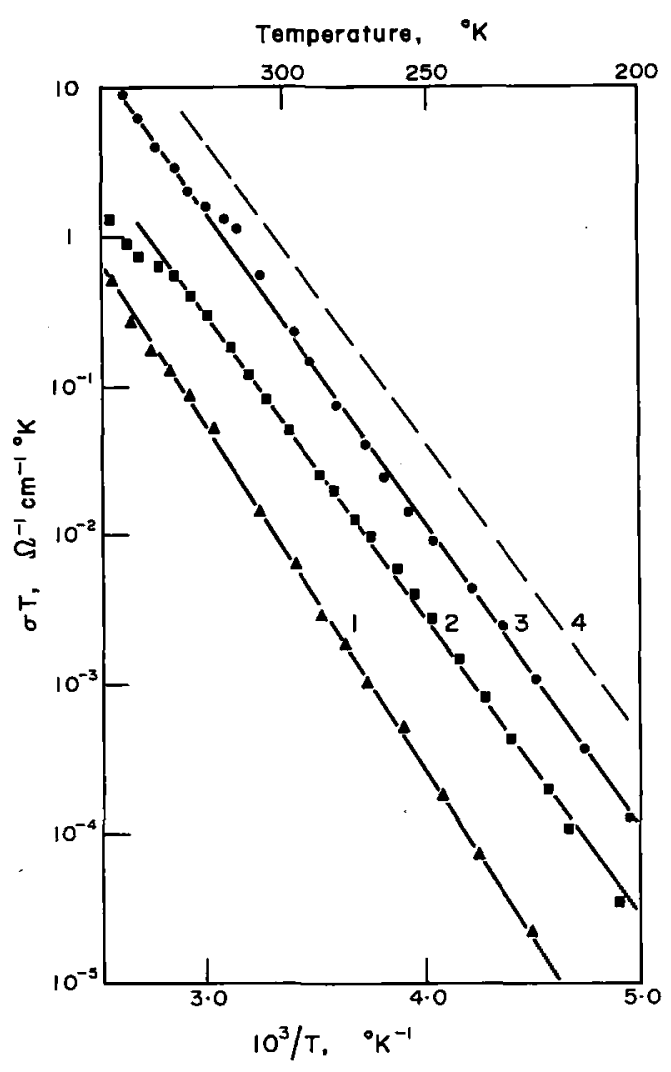

Fig. 3. Temperature dependence of ionic conductivity for a typical selection of AgI layers deposited on mica. Layer thickness (1) $1800 \AA$; (2) $4300 \AA$; (3) $100 \AA$;

(4) represents an activation energy of $0.4 \mathrm{eV}$.

The ionic conductivity results in Fig. 2(a) illustrate that both nominally pure and copper doped single crystals with graphite electrodes display two distinct linear regions of similar slope. On the other hand, while nominally pure single crystals with silver electrodes have the same high temperature characteristics, the low temperature region is markedly different. Since all specimens tested with the current flowing parallel to the $c$-axis showed the same elevated temperature characteristics it seems reasonable to identify this portion of the curves as the intrinsic or structure insensitive region.

It is proposed that the low temperature regime for nominally pure crystals with graphite electrodes can be associated with the motion of excess $\mathrm{Ag}^{+}$vacancies. The addi- tional $\mathrm{Ag}^{+}$vacancies are presumably generated when $\mathrm{Ag}^{+}$ions are electrolytically deposited during the electrode conditioning process.

The above proposal is supported by the low temperature results for a crystal doped with $\mathrm{Cu}^{2+}$ ions, where nominally the addition of each $\mathrm{Cu}^{2+}$ ion creates one $\mathrm{Ag}^{+}$vacancy. Normally little significance could be placed on the results from a single specimen; however, in the present context where the results for the copper doped crystal are only used in support of the other data the authors feel this is justified.

The results for nominally pure specimens with silver electrodes can be reconciled by attributing the low temperature regime to an increase in the interstitial $\mathrm{Ag}^{+}$ion concentration. The slight non-stoichiometry is assumed to be created by diffusion of silver from the electrodes.

In the intrinsic region $E_{1}=\frac{1}{2} W+U$ and, since two possible values of $U$ are available from $E_{21}$ and $E_{22}$, two possible $W$ values can be derived on the alternative assumptions that it is interstitials or vacancies which are the dominant carriers in the intrinsic region and $c$-axis direction. The former assumption gives the unlikely low value of $0.34 \mathrm{eV}$ for $W$ and we therefore accept the latter view and the $W$ value of $0.60 \mathrm{eV}$, which is in better agreement with the other determinations shown in Table 2.

Figure 2(b) shows results for nominally pure crystals with aquadag electrodes and current flow normal to the $c$-axis. The use of aquadag electrodes implies that the low temperature region is associated with the motion of $\mathrm{Ag}^{+}$vacancies. Unfortunately we have no measurements on $\mathrm{Cu}^{2+}$ doped crystals in the $a$ direction to yield direct confirmation of these assumptions. In the intrinsic region $E_{1}{ }^{a}=\frac{1}{2} W+U$ and, since the value of $W$ is independent of the current direction, $U$ may be determined from $E_{1}{ }^{a}$. However, the value of $U$ calculated this way is considerably less than the value determined from the extrinsic 
region (where $E_{2}{ }^{a}=U$ ), consequently conductivity in the intrinsic region must be associated with the motion of interstitial $\mathrm{Ag}^{+}$ions.

Accepting these interpretations and using the experimentally determined activation energies shown in Table 1 , where superscript $c$ refers to measurements in the $c$-axis direction and $a$ to measurements normal to this direction, we derive the conclusions set out in the first line of Table 2. Here subscript + and - refer to $\mathrm{Ag}^{+}$interstitials and $\mathrm{Ag}^{+}$ vacancies respectively.

Also shown in Table 2 are such values of these fundamental activation energies as can be derived from other work. Since these measurements were all on polycrystalline samples, some of the interpretations are uncertain and all have been derived in a variety of ways. The values of $U^{c}$ and $U^{a}$ ascribed to Jordan and Pochon [19], for example, are actually activation energies for diffusion of silver in hexagonal $\beta \mathrm{AgI}$ and cubic $\gamma \mathrm{AgI}$ measured by a radioactive tracer technique. Lieser's value of $W$ came from calorimetric measurements.

Table 1.Experimentally determined activation energies (all in $\mathrm{eV}$ )

\begin{tabular}{ll}
\hline$E_{1}^{c}$ (all crystals) & $0.79 \pm 0.02$ \\
$E_{2}^{c}\left(\mathrm{Cu}^{2+}\right.$ doped) & $0.49 \pm 0.02$ \\
$E_{21}^{c}$ ('pure crystals', graphite electrodes) & $0.50 \pm 0.03$ \\
$E_{22}^{c}$ ('pure crystals', silver electrodes) & $0.62 \pm 0.02$ \\
$E_{1}^{a}$ ('pure crystals') & $0.59 \pm 0.02$ \\
$E_{2}^{a}$ ('pure crystals' graphite electrodes) & $0.39 \pm 0.02$ \\
\hline
\end{tabular}

where values are shown spread between two or more columns, it is not clear from the nature of the experiment to which column they should be assigned, though the numerical result itself sometimes makes this obvious. The value of $U$ given by Lieser [21] does not represent a direct measurement, but relies upon the assumption that his measured $E$ is in fact $E_{1}$.

The only other reported measurements on single crystals of $\beta$ AgI are those of Lakatos and Lieser[12] who found, for both axial directions, not two exponential conductivity segments but rather a continuously changing activation energy which increased with increasing temperature. The reason for this behaviour is not apparent.

It is noteworthy that the activation energies $U_{ \pm}^{c}$ for diffusion in the $c$-axis direction are considerably greater than those for diffusion normal to this direction, $U_{ \pm}{ }^{a}$. It is easy to interpret this qualitatively since the wurtzite structure may be thought of in terms of alternating layers of positive and negative ions, each layer lying normal to the $c$-axis.

The present study indicates that for intrinsic material the conductivity can be attributed to the motion of silver ion vacancies in the $c$-axis direction and silver ion interstitials normal to this direction. It is not possible to conclude from these measurements alone if an interstitialcy type mechanism should be invoked, similar to that used to explain intrinsic conductivity in the other silver halides $[15,24]$. The intrinsic conductivity

Table 2. Fundamental activation energies for $\beta \mathrm{AgI}$ (all in $\mathrm{eV}$ )

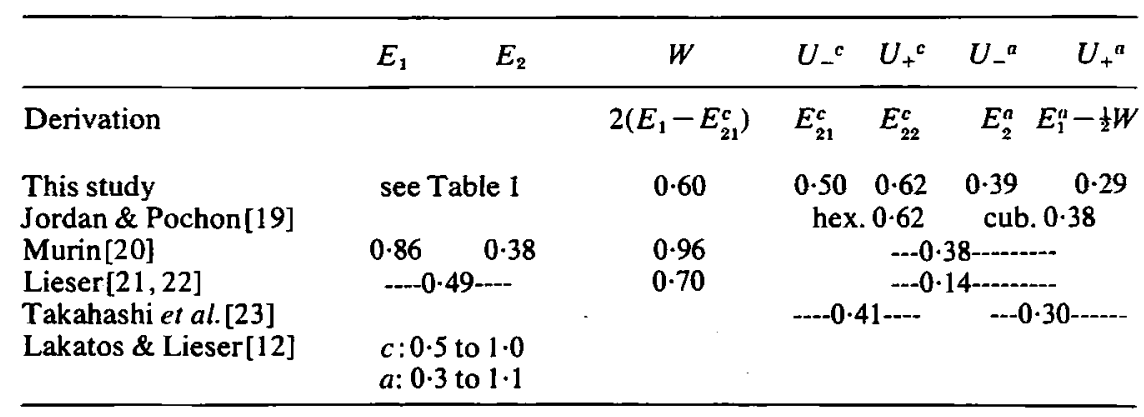


parallel and normal to the $c$-axis direction can be summarized by the following equations, ( $\sigma$ measured in $\Omega^{-1} \mathrm{~cm}^{-1}$ and $k T$ in $\mathrm{eV}$ )

$$
\begin{aligned}
\sigma_{\|} & =8.8 \times 10^{5} \exp (-0.79 / k T) \\
\sigma_{\perp} & =5.7 \times 10^{2} \exp (-0.59 / k T) .
\end{aligned}
$$

Turning now to thin films, we find a variety of conductivity characteristics, a representative selection of which are shown in Fig. 3. Electron diffraction studies $[1,10]$ have shown that the films used are well oriented with the stacking direction normal to the substrate, so that it is relevant to compare the results with those for single crystals measured in the $a$-direction. Because of the large surface-tovolume ratio of thin films, it is reasonable to expect extrinsic behaviour and, indeed, the slope of the plots is quite close to the value $U_{-}{ }^{a}=0.39 \mathrm{eV}$ expected from the single crystal results, if the majority carriers are silver ion vacancies rather than excess silver ions. There is little to be gained at present by any more detailed discussion of the curves. It is relevant to note, however, that the thinfilm results show several points of similarity with those obtained on powder compacts $[20,25]$, which is, of course, expected because of the large surface-to-volume ratio in each case.

\subsection{Experimental}

Specimens approximately $0.15 \mathrm{~cm}$ long were cut from hexagonal prism-shaped single crystals grown from KI-AgI solution. Some specimens were mounted directly between two brass electrodes, while others first had Aquadag electrodes applied. Edge effects were reduced by arranging for the specimen to be smaller in diameter than the electrodes by at least twice its thickness. All of the six specimens tested over part or all of the temperature $\left(-80\right.$ to $\left.+65^{\circ} \mathrm{C}\right)$ and frequency $(20 \mathrm{~Hz}$ to $100 \mathrm{kHz})$ ranges, had the electric field applied parallel to the $c$-axis. Capacitance and loss measurements were made with a General Radio 1620A Capacitance Measuring Assembly which allowed accurate determination both of the real part $\epsilon^{\prime}$ of the dielectric constant and of the loss factor $\tan \delta$. The amplitude of the alternating voltage applied across the specimen was $20 \mathrm{~V}$ peak-to-peak for all frequencies. Before testing specimens of $\beta \mathrm{AgI}$, the assembly was checked over the complete temperature and frequency range using a specimen of polytetrafluoroethylene for which the dielectric properties are well known.

Measurement of the real part $\epsilon^{\prime}$ of the dielectric constant is estimated to be correct to better than \pm 2 per cent for $\epsilon^{\prime}<20$. At low frequencies $(<1 \mathrm{kHz})$ and large values of $\epsilon^{\prime}(>500)$ the accuracy decreases to \pm 10 per cent.

\subsection{Results and discussion}

Typical plots of $\tan \delta$ against frequency are shown in Fig. 4 and corresponding plots for

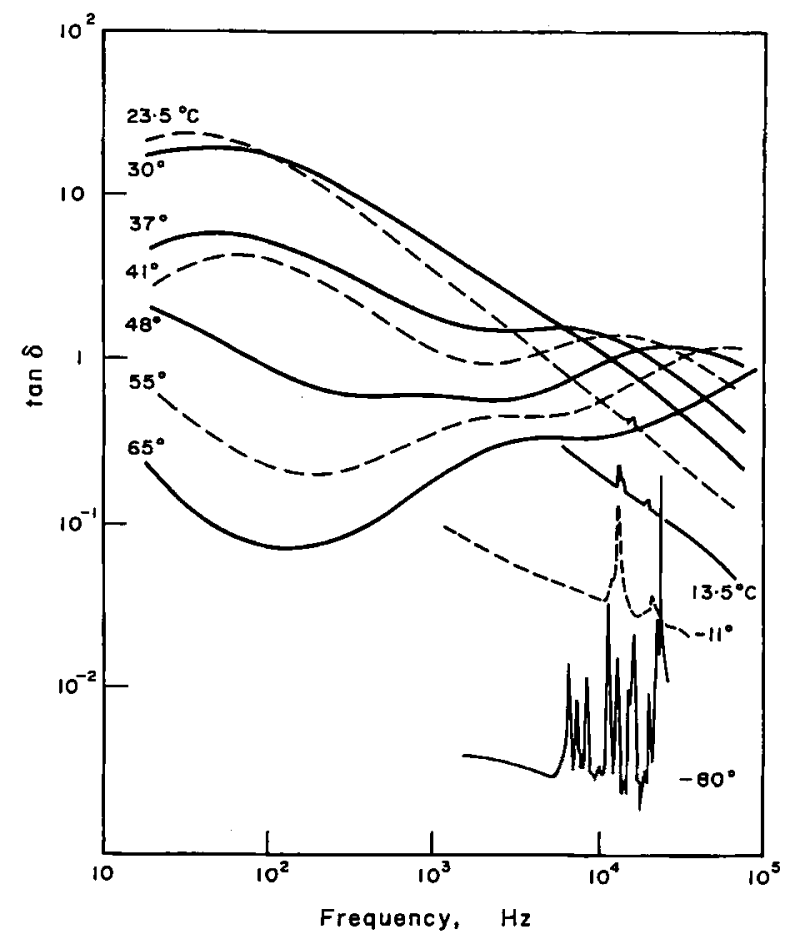

Fig. 4. Variation of the loss tangent with frequency in $\beta$ AgI. 


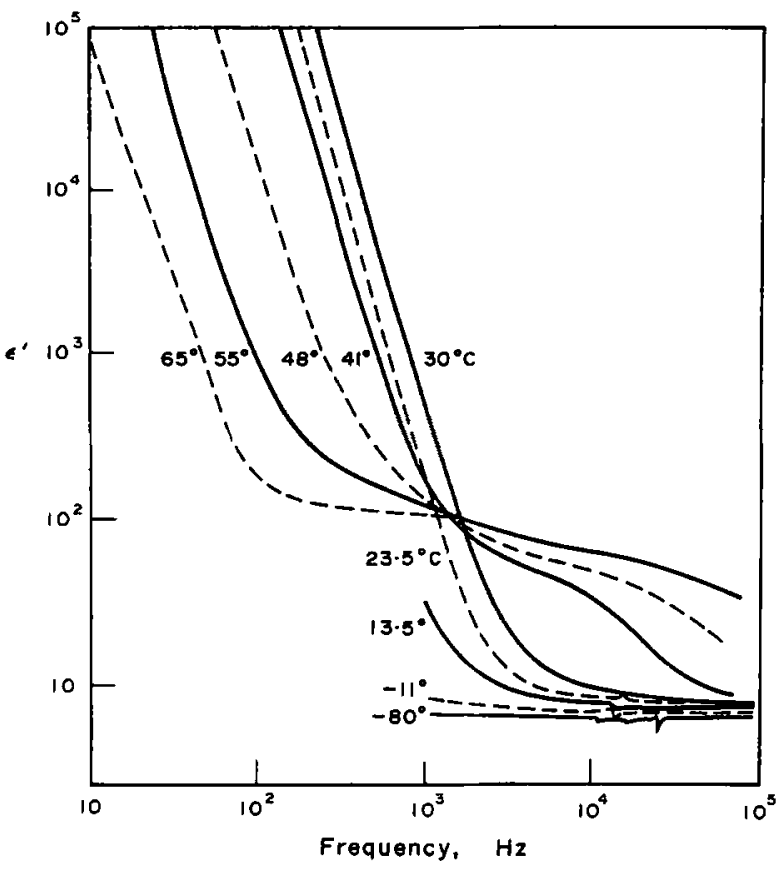

Fig. 5. Variation of the apparent dielectric constant $\epsilon^{\prime}$ of $\beta \mathrm{AgI}$ with frequency.

$\epsilon^{\prime}$ in Fig. 5. When a specimen was held at a constant temperature above about $50^{\circ} \mathrm{C}$, values of $\tan \delta$ and $\epsilon^{\prime}$ increased with time. The effect became more pronounced as the temperature increased until, at about $90^{\circ} \mathrm{C}$, it was impossible to balance the bridge. On cooling the specimen to room temperature, the original values of $\tan \delta$ and $\epsilon^{\prime}$ were again obtained.

From Figs. 6 and 7 it is clear that the static dielectric constant at low temperatures, when space-charge effects are absent, has a value in the range 6 to 8 , the precise result at $-60^{\circ} \mathrm{C}$ being $6 \cdot 8 \pm 0 \cdot 2$. This result agrees well with the value $7 \cdot 0 \pm 0.3$ reported by Bottger and Geddes [26] from measurements on powdered samples at $27^{\circ} \mathrm{C}$ in the frequency range $10^{6}$ to $10^{7} \mathrm{~Hz}$ but differs markedly from the results of Sathe et al. [27] who give a value of 33.86 from measurements on powder samples under unspecified conditions. Presumably in this latter case space charge effects were present.

The most reasonable interpretation of the

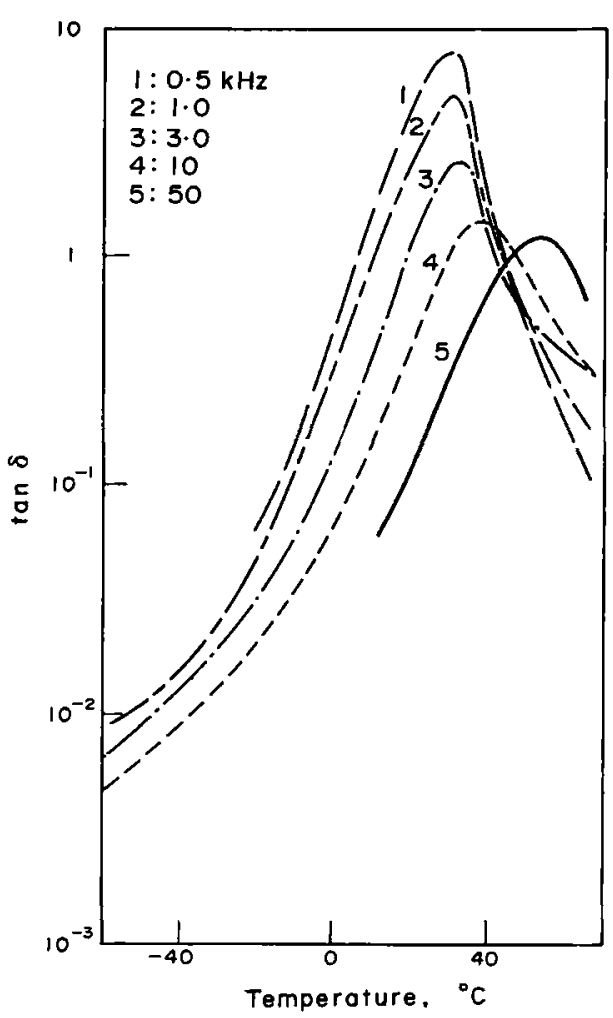

Fig. 6. Temperature dependence of the loss angle in $\beta A g I$ derived from the results given in Fig. 4.

behaviour shown in Figs. 6 and 7 is that, at low frequencies and high temperatures, ionic migration takes place and increases the measured dielectric constant $\epsilon^{\prime}$ by the creation of space-charge layers at the electrodes.

Another interesting feature of the curves shown in Fig. 4 is the loss peak shown in the frequency range $10^{4}-10^{5} \mathrm{~Hz}$ for temperatures above about $37^{\circ} \mathrm{C}$. This is a rather broad peak which shifts steadily to higher frequencies as the temperature is raised, in the same way as does a simple Debye relaxation peak. A second somewhat similar peak appears in the frequency range $10^{3}-10^{4} \mathrm{~Hz}$ for temperatures above about $50^{\circ} \mathrm{C}$.

There seem to be three possible mechanisms which may be invoked to explain the observed relaxation spectra:

(i) Aliovalent impurities 
(ii) Interfacial polarization

(iii) Reorientation of Frenkel defects.

Restricting our attention, for the moment, to the peak of higher frequency, we find that its shift with frequency implies an activation energy of $0.84 \pm 0.05 \mathrm{eV}$. The magnitude of the peak above the interpolated base line provided by the conductivity losses increases with increasing temperature, which implies that the concentration of dipoles causing the relaxation also increases with temperature. At $55^{\circ} \mathrm{C}$ the value of $\tan \delta_{\max }$ due to this loss mechanism is about 1 at a frequency of 50 $\mathrm{kHz}$ which, using the usual Debye treatment [28] as an approximation, implies a concentration $n$ of contributing dipoles, each of moment $\mu$, such that $n \mu^{2} \sim 10^{-32} \mathrm{C}^{2} \mathrm{~m}^{-1}$. For a typical ionic dipole having a length of

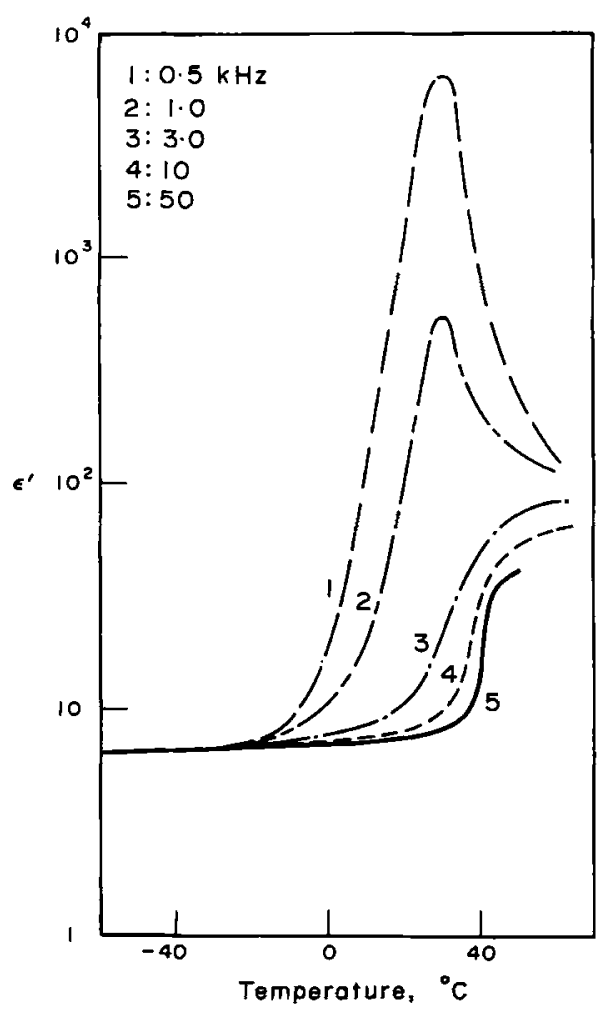

Fig. 7. Temperature dependence of the apparent dielectric constant $\epsilon^{\prime}$ in $\beta \mathrm{AgI}$, derived from the results given in Fig. 5. the order of a lattice spacing, this leads to a dipole moment $\mu \sim 10^{-28} \mathrm{Cm}$, which implies a dipole density $n \sim 10^{24} \mathrm{~m}^{-3}$, compared with the total ion density $\sim 10^{28} \mathrm{~m}^{-3}$ in AgI. This concentration of dipoles is about two orders of magnitude greater than any expected aliovalent impurity ion and about an order of magnitude greater than the measured potassium impurity.

Consider now the second possibility, interfacial polarization. Effects of this type have been observed in $\mathrm{NaCl}$ by Wimmer and Tallan[29] and by Kessler and Moriani[30], while similar effects have been observed in $\mathrm{AgBr}$ and explained theoretically by Friauf [31]. The present results for the loss peak of higher frequency show a marked similarity to the relaxation spectra of $\mathrm{NaCl}$ given by Wimmer and Tallan. In addition the activation energy of $0.84 \mathrm{eV}$, characteristic of ionic conduction in the region, suggests this peak is most probably caused by blocking at the electrodes. There is, however, still the problem of the second peak which it is difficult to explain by this argument.

The third possibility would involve the reorientation of vacancy pairs, which as pointed out by Greenwood [16] should involve two relaxation peaks in the case of Frenkel defects (i) normal lattice ion moving into a vacancy (ii) interstitial ion moving into a vacancy. At $55^{\circ} \mathrm{C}$ the apparent concentration of dipoles is of the same order of magnitude as the concentration of Frenkel defects, as calculated from the value of $W$ given in Table 2. The occurrence of the second peak with an activation energy of $0.63 \pm 0.03 \mathrm{eV}$ tends to support this picture. There are, however, difficulties associated with such an interpretation. The activation energy involved in the relaxation should be that associated with the diffusion process alone which, from Table 2 is $0.50 \mathrm{eV}$ for vacancies and $0.62 \mathrm{eV}$ for interstitials, the $c$-axis direction being the relevant one in the present context. Neither of these energies is very close to the measured $0.84 \mathrm{eV}$ for the higher frequency peak, 
although the lower frequency peak (0.63 $\mathrm{eV}$ ) could well be associated with the motion of interstitial silver ions.

Finally we note the very sharp absorption peaks shown at low temperatures in Fig. 4. The corresponding behaviour of $\epsilon^{\prime}$ in this range, as shown in Fig. 5, suggests that some sort of resonance phenomenon is being observed. To ensure that these peaks were not spurious, it was checked that they were not present when the specimen was replaced by a similarly sized block of $\mathrm{NaCl}$, Calcite, Polythene or Teflon, their absence in these cases confirming them to be a genuine property of AgI.

Since the crystal structure of $\beta$ AgI lacks a centre of symmetry, the natural interpretation is that these peaks arise from some piezoelectric resonance phenomenon. This conclusion is reinforced by the failure to observe the peaks in a differently prepared crystal with the field normal to the $c$-axis direction. The expected resonance frequency for a crystal of the size used is, however, around $400 \mathrm{kHz}$ rather than the $10 \mathrm{kHz}$ observed. Tests with a different sample holder reduced the probability that we were observing an incidental resonance of the apparatus excited by the crystal, but the position is not yet clear.

Acknowledgements - This work is part of a programme supported by the Australian Research Grants Committee and, in its earlier stages, by the C.S.I.R.O. Division of Radiophysics. One of us (G.C.) is grateful to the University of New England for a Teaching Fellowship, during tenure of which the work was done. It is also a pleasure to express our thanks to the technical staff of the Department for their valuable assistance.

\section{REFERENCES}

1. COCHRANE G., Ph.D. Thesis, University of New England 1968 (unpublished).

2. BURLEY G.,Am. Mineral. 48, 1266 (1963).
3. BLOCH R. and MOLLER H., Z. Phys. Chem. A152, 245 (1931).

4. SCHNEER C. J. and WHITING R. W., Am. Mineral. 48, 737 (1963).

5. DAVIS B. L. and ADAMS L. W., Science 146, 519 (1964).

6. MAJUMDAR A. J. and ROY R., J. Phys. Chem. 63, 1858 (1959).

7. KOLKMEIJER N. H. and HENGEL J. W. A., Z. Kristallogr. 88, 317 (1934).

8. CORRIN M. L. and STORM N. S., J. Phys. Chem. 67, 1509 (1963).

9. JOST W. and OEL H. J., Disc. Faraday Soc. 23, 137 (1957).

10. COCHRANE G.,J. Cryst. Growth. 7, 109 (1970).

11. COCHRANE G., Brit.J. appl. Phys. 18, 687 (1967).

12. LAKATOS E. and LIESER K. H., Z. Phys. Chem. 48, 228 (1966).

13. MILLS, D. R., PERROTT C. M. and FLETCHER N. H., J. Cryst. Growth 6, 266 (1970).

14. HALBERSTADT E. S., Nature Lond. 216, 574 (1967).

15. LIDIARD A. B., Handbuch der Physik, Vol. XX, 246 Berlin, (1957).

16. GREENWOOD N. N., Ionic Crystals Lattice Defects and Nonstoichiometry, Butterworths, London (1968).

17. JOST W. and NOLTING J., Z. Phys. Chem. 7, 383 (1956).

18. TOLANSKY S., Multiple Beam Interferometry of Surfaces and Films, Clarendon Press, Oxford (1949).

19. JORDAN P. and POCHON M., Helv. Phys. Acta. 30, 33 (1957).

20. MURIN A. N., LUR'E B. G. and TARLAKOV Yu P., Fiz. tverd. Tela 3, 3299 (1961).

21. LIESER K. H., Z. Phys. Chem. NF9, 302 (1956).

22. LIESER K. H., Z. Phys. Chem. NF2, 238 (1954).

23. TAKAHASHI T., KUWABARA K. and YAMAMOTO O., J. electrochem. Soc. 116, 357 (1969).

24. FRIAUF R. J., Phys. Rev. 105, 843 (1957).

25. MRGUDICH J. N., J. electrochem. Soc. 107, 475 (1960).

26. BOTTGER G. L. and GEDDES A. L., J. chem. Phys. 46, 3000 (1967).

27. SATHE N. V., PHALNIKAR N. L. and BHIDE B. V.,J.Ind. Chem. Soc. 22, 29 (1945).

28. BRECKENRIDGE R. G., J. chem. Phys. 16, 959 (1948).

29. WIMMER J. M. and TALLAN N. M., J. appl. Phys. 37, 3728 (1966).

30. KESSLER A. and MARIANI E., J. Phys. Chem. Solids 29, 1079 (1968).

31. FRIAUF R. J,,J.chem. Phys. 22, 1329 (1954). 